\title{
Comparative analysis of lactaptin produced in bacterial and eukaryotic cells. Purification and activity
}

O. Chinak ${ }^{1 *}$, O. Volkova 2 , T. Belovezhec 2 , A. Gorchakov², A. Tkachenko ${ }^{1}$,

E. Kuligina ${ }^{1}$, V. Ricter ${ }^{1}$, O. Koval ${ }^{1,3}$

${ }^{1}$ Institute of Chemical Biology and Fundamental Medicine, SB, RAS, Novosibirsk, Russia

${ }^{2}$ Institute of Molecular and CellularBiology SB RAS, Novosibirsk, Russia

${ }^{3}$ Novosibirsk State University, Novosibirsk, Russia

*e-mail: chinakolga@gmail.com

Key words: lactaptin, casein, anticancer protein, affinity chromatography

Motivation and Aim: Lactaptin is a fragment of human milk $\kappa$-casein, it has been previously shown to induce the apoptosis of cancer cells in culture with no cytotoxic activity toward non-malignant cells. Its recombinant analogue (RL2) produced in E.coli demonstrated tumor growth suppression in vivo. Given that correct folding of recombinant human proteins occurs in eukaryotic cells we constructed new recombinant plasmid for expression lactaptin analog EL1 (eukaryotic lactaptin 1) in human cells. Besides a leader sequence for secretion, recombinant lactaptin contained hexahistidinetag. Recombinant lactaptin EL1 production, secretion, harvesting and purification were in the focus of our work.

Methods and Algorithms: EL1production and secretion by HEK293T cells was detected by Western-blot analysis in cell lysates as well as in cultural medium. To purify EL1 we used cation-exchange and immobilized metal ion affinity chromatography (IMAC).

Results: IMAC is a commonly used protein purification procedure. However, enrichment of eukaryotic cell culture medium with histidine and cysteine results in competitive binding of these amino asids with immobilized metal. Moreover, we found that numerous histidine-containing serum proteins contaminated EL1 eluates. To overcome these difficulties we performed cation-exchange chromatography as an initial step. The chromatography protocol (resin, ionic concentrations and $\mathrm{pH}$ ) was optimized to remove free histidine and proteins with similar retention properties from the EL1-containing solutions. Using IMAC chromatography for subsequent EL1 purification we obtained the protein with up to $95 \%$ purity.

Conclusion: Detailed protocol for recombinant lactaptin EL1 purification was developed. Acknowledgements: Supported by the by the Russian Ministry of Education and Science, Agreement 14.604.21.0169 (unique project identifier RFMEFI60417X0169). 